\title{
INFECTIOUS AORTITIS IN A PATIENT WITH ANKYLOSING SPONDYLITIS UNDERGOING IMMUNOBIOLOGICAL THERAPY
}

Fábio Batistella1,*, Larissa Baroni ${ }^{1}$, Anna Laura Ribeiro Ribas ${ }^{1}$, Jalila Duarte Lugo Xavier da Rosa ${ }^{1}$, Laura Faistel Marques ${ }^{1}$, Daniel Fransosi Marques ${ }^{1}$, Bernardo Lucca Eisenreich ${ }^{1}$, João Vítor Rigo Pontel ${ }^{1}$, Tamiris Natália Chiossi ${ }^{1}$, Júlia Minosso ${ }^{1}$, Thaís Lubian ${ }^{1}$, Jaqueline Missiaggia ${ }^{1}$, Bruna Santin Andreis ${ }^{1}$, Josmar Ramon Kruger Klock ${ }^{1}$, Gabriel Karpowicz ${ }^{1}$, Caroline Arais Schmidt ${ }^{1}$, Eduardo Gracioli ${ }^{1}$, Matheus Augusto Eisenreich ${ }^{1}$, Mateus Picada Correa ${ }^{1}$

1.Universidade de Passo Fundo, Passo Fundo (RS), Brazil.

*Corresponding author: fabiobatistella@upf.br

\section{BACKGROUND}

The immunobiological therapy provides symptomatic and quality of life improvement in patients with ankylosing spondylitis (AS). Patients undergoing this therapy should be monitored regularly as they are at increased risk for infectious diseases. Infectious aortitis is a rare clinical condition with a challenging diagnostic definition. In this paper our objective is describe a case of subacute abdominal and testicular pain in an infliximab treated AS patient, whose investigation prove to be Salmonella aortic infection.

\section{CASE REPORT}

Male patient, 74 years old, diagnosed with AS diagnosed 16 years ago, Diabetes mellitus, benign prostatic hyperplasia. Using infliximab $400 \mathrm{mg}$ every 12 weeks, dutasteride plus tamsulosin and metformin. Admitted at emergency room with non-continuous low abdominal and testicular pain and intermittent fever for 6 weeks. Received treatment with ceftriaxone and moxifloxacin prior to admission, without improvement. Described pain relief with the use of naproxen. Two days before admission, the pain and fever $\left(39^{\circ} \mathrm{C}\right)$ worsened. In the initial assessment at emergency department, the patient was in good general condition, afebrile, hemodynamically stable. Cardiac auscultation presented aortic murmur. Abdominal and testicular examination was normal. Proceed with hospitalization for investigation. The patient presented with new episodes of intense abdominal and testicular pain. The complementary exams showed C-reactive protein $34(<6)$, testicular ultrasound with varicocele, transesophageal echocardiography without criteria for endocarditis, normal thoracic tomography and Abdominal computed tomography with abdominal aorta showing a parietal ulcer with 13mm of diameter. Blood cultures were negative. Initial antibiotic therapy with piperacillin-tazobactam with later adjustment to linezolid and meropenem. After 4 days of new antibiotic therapy and partial symptomatic control, he developed an acute abdomen due to aortic rupture and underwent surgical intervention. The patient died two days later. Culture of material collected in the surgery (aortic wall) was performed with growth of Salmonella spp.

\section{CONCLUSION}

The diagnosis of infection in immunosuppressed patients is a constant challenge in rheumatology, especially under conditions of atypical presentation. Infectious aortitis is a serious disease with high morbidity and mortality. Previous arterial aneurysm, advanced age, trauma and immunosuppression are some risk factors for its development. The diagnosis is based upon imaging and confirmed by culturing and organism from the blood an or from the aortic wall. Salmonella is the pathogen identified in $15-24 \%$ of diagnoses. The treatment consists in antibiotic therapy and surgery in selected cases.

\section{KEYWORDS}

Aortitis, Ankylosing spondylitis, Immunobiological therapy. 